\title{
A Disposable Intravaginal Device for the Management of Stress Urinary Incontinence
} \author{
and Bernard Hertzman ${ }^{5}$ \\ ${ }^{I}$ The Procter \& Gamble Company, Winton Hill Business Center, Cincinnati, Ohio, USA \\ ${ }^{2}$ CTI Clinical Trial \& Consulting Services, Inc, Cincinnati, Ohio, USA \\ ${ }^{3}$ Advanced Urogynecology, Cincinnati, Ohio, USA \\ ${ }^{4}$ Drs Katz, Kade, and Hewitt, Inc. Cincinnati, Ohio, USA \\ ${ }^{5}$ The Urology Group, Cincinnati, Ohio, USA
}

Miranda A. Farage*,1, William S. Aronstein ${ }^{2}$, Kenneth W. Miller ${ }^{1}$, Mickey Karram ${ }^{3}$, Molly Katz ${ }^{4}$

\begin{abstract}
Urinary incontinence affects many women over the age of 50 with a substantial detrimental effect on daily activities and the quality of life. Surgical treatments are invasive, expensive, and not always successful. Patients are also treated with a variety of nonsurgical therapies (physical therapy, pharmaceutical interventions, and various vaginal and urethral inserts), but these have poor compliance rates and limited efficacy in patients with mild to moderate stress urinary incontinence (SUI). Incontinence pessaries have demonstrated efficacy in the treatment of SUI but can cause vaginal erosion, foul vaginal odors, and other complications associated with their prolonged residence in the vagina.

A novel disposable intravaginal device, which can be worn during active hours and then discarded, was evaluated in this study for safety, ease of use, and efficacy in the treatment of SUI. Fifty-seven women with an average of one episode of SUI per day were fitted with the device, allowed to acclimate to wearing it during the day, and then asked wear the device and pre-weighed incontinence pads for 12 hours a day.

Use of this device was significantly associated with a statistically significant decrease in SUI episodes, a decrease in unintentional urine output, a decrease in self-reported bladder control difficulty, and a self-reported improvement in quality of life. Subjects rated the comfort of device use during the fitting period, as well as during later device usage. No serious adverse events were reported. Results show that the intravaginal device is safe, relatively comfortable, and effective at reducing the frequency and psychosocial impact of SUI.
\end{abstract}

Keywords: Intravaginal device, pad weight gain, pessary, stress urinary incontinence.

\section{INTRODUCTION}

Urinary incontinence imposes a psychosocial burden on its sufferers, significantly impacting self- confidence, social interaction, and overall quality of life (QoL) [1]. and also has a major economic impact on society [2]. Incontinence affects women at substantially higher rates than men. A survey of ambulatory men (mean age 50) found the prevalence of urinary incontinence to be $12.7 \%$ [1]. While a similar study found the prevalence in women (mean age 50) to be $53.2 \%$, more than four times higher [2]. The risk of incontinence in women increases with age [3], reaching, in the study above, $76.1 \%$ in women in their sixth decade of life [2].

The cost of treating urinary incontinence reached 19.5 billion dollars in the year 2000 [4]. Stress urinary incontinence (SUI), defined as a loss of urine without active bladder contraction, is the most common type of urinary incontinence (afflicting $78 \%$ of incontinent women) [5] and is

*Address correspondence to this author at the Procter and Gamble Company, Feminine Care Innovation Center, Winton Hill Business Center, 6110 Center Hill Road, Box 136, Cincinnati, OH 45224, USA; Tel: + 1513 634 5594; Fax: + 1 (866) 622-0465; E-mail: farage.m@pg.com typically induced by sneezing, coughing, lifting or exercise [6]. Surgical treatments are largely reserved for severe SUI. Nonsurgical therapies include basic urine collection products, physical therapy and behavior modification, pharmaceutical agents, and vaginal and urethral inserts. However, some of these have had limited efficacy in the treatment of mild to moderate SUI, have poor compliance rates, are associated with various adverse events (AEs) [7], and often lack patient satisfaction [8]. Therapy alternatives for women with SUI, particularly those who are not suitable candidates for or who wish to avoid surgery, are of significant potential benefit.

A disposable intravaginal device designed for the temporary management of urinary stress incontinence, the TIPI intravaginal device, is manufactured by ConTIPI Ltd (Caesarea, Israel). The device is comprised of a resin core which provides tension-free support whenever pressure is transferred from the abdominal cavity to the pelvic floor [7]. The core is covered by a soft biocompatible cover of nylon mesh; both core and cover are contained within a smooth, smalldiameter applicator similar to those used for tampon insertion [7]. This single use, disposable, intravaginal device is 
inserted by the patient (in a manner analogous to the insertion of a tampon).

A previous study conducted among 60 women diagnosed with severe SUI (who would otherwise have been scheduled for surgery) evaluated device efficacy by measuring unintentional urine output. In addition to the device, subjects wore pads which were collected and weighed. Of the 50 women who wore the intravaginal device as well as collection pads for the entire 28-day test period, $94 \%$ achieved a $\geq 70 \%$ reduction in pad weight gain $(\mathrm{PWG})(\mathrm{P}<0.001)$. In addition, a retrospective questionnaire-based assessment of satisfaction with the device demonstrated a high degree of user satisfaction with the device, with a mean satisfaction score of 29.72 out of a total of 33 points [7].

A concomitant research study evaluated patientperceived efficacy of the TIPI intravaginal device by two validated questionnaires. Use of the device was associated with substantial improvement in QoL after 28 days of device usage as compared to a prestudy evaluation. The Incontinence Impact Questionnaire [IIQ-7] observed a decrease in the impact of urinary incontinence on QoL from $41.8( \pm 24.1)$ to $4.4( \pm 8.7$. ( $\mathrm{P}<0.001)$. The Urogenital Distress inventory [UDI-6]) also found a decrease in distress, with the mean total score dropping from $48.2( \pm 16.1)$ to $11.5( \pm 11.9, \mathrm{P}<0.001)[8]$

The present study evaluated real-life use by following women who wore the device for up to 12 hours per day, and was designed to evaluate the safety, efficacy, and tolerability of the TIPI intravaginal device as well as a new applicator design. Device safety was evaluated by physical examination, gynecological assessments including Pap smear and vaginal $\mathrm{pH}$, surveillance for vaginal or urinary infection, and assessment of any other AEs. Efficacy was assessed by reduction in SUI episodes, reduction in PWG, and subjective evaluation of patients' perception of their bladder condition. Overall effect of the device on incontinence-related QoL was also assessed.

\section{MATERIALS AND METHODOLOGY}

This study was an open-label, uncontrolled study of 57 women in the Cincinnati, Ohio, area. Women were screened for study eligibility at the initial visit after providing written informed consent at the initial visit. The protocol, informed consent form, and any information provided to the patients were reviewed and approved by an independent Institutional Review Board (IRB) and the study was conducted according to Investigational Device Exemption (IDE) requirements. Study conduct followed established and accepted Good Clinical Practice (GCP) guidelines prescribed by the FDA. The study was monitored by the Sponsor (Procter \& Gamble Company) to verify compliance with the GCP guidelines.

Eligible patients were between 18 and 70 years of age, with a history of at least seven episodes of SUI per week prior to entering the study. They were also required to have used tampons for monthly periods and be willing to comply with all study requirements, including the use of the TIPI intravaginal device to control SUI. Patients were excluded if they were pregnant or trying to get pregnant, had vaginal abnormalities or a history of vaginal delivery or surgery in last 3 months, had a history of toxic shock symptoms or difficulty with the use of intravaginal devices (including tam- pons), had a history of incontinence surgery or irradiation therapy, were prone to recurrent vaginal infection or urinary tract infections (UTIs), or had used anticoagulants in the last 30 days.

The following procedures were carried out on prospective subjects during the first (screening) visit: complete history and physical examination (including a gynecological evaluation with Pap smear and vaginal $\mathrm{pH}$ ), a vaginal swab for assessment of vaginal infection and sexually transmitted diseases (STDs), and a pregnancy test. Subjects also completed a validated QoL questionnaire the IIQ-7, which evaluated patient perception of QoL, and the Patient Perception of Bladder Condition (PPBC) questionnaire, which evaluated patient perception of their bladder condition.

Following the screening visit, eligible women were instructed to wear pre-weighed pads for 12 hours each day during a 5-day baseline period and to record the number and type of incontinence episodes experienced during each 12hour period. The 5-day baseline period was followed by a $7-$ day fitting period during which the patient identified the best fit among a selection of three device sizes and then acclimated to device usage. Women self-fit the device by inserting the device size recommended by the instructor and then either increased or decreased the device size until they felt that they had determined the size that provided optimal prevention of leakage as well as comfort. Patients had ongoing access to all available sizes during the fitting process (Fig. 1).

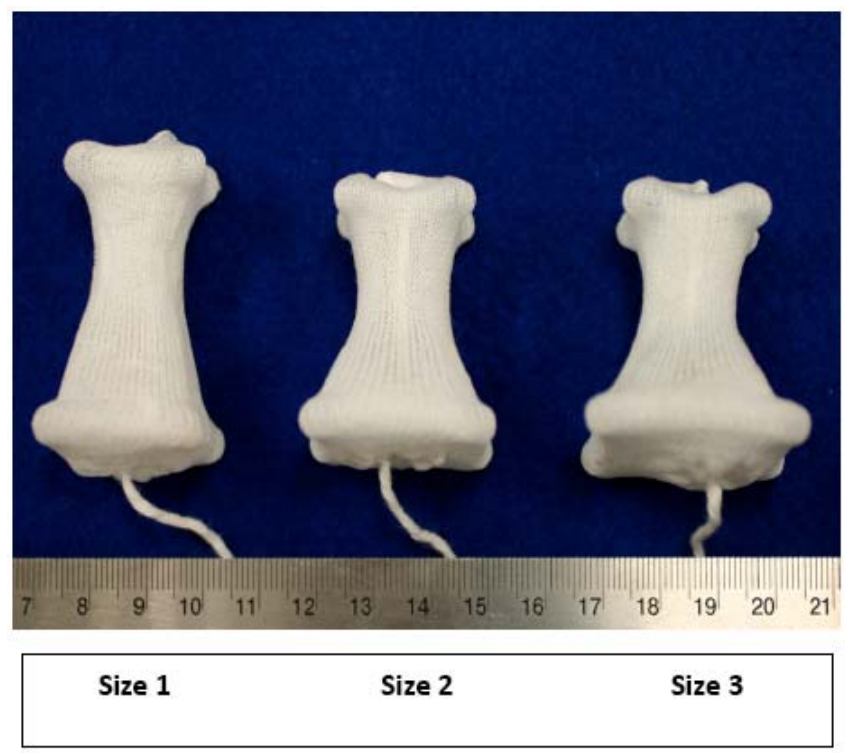

Fig. (1). Three available sizes of TIPI intravaginal devices for urinary stress incontinence.

The fitting period was followed by 14 days of device usage in which the previously selected device size was used for up to 12 hours daily. Daily device usage was extended from 8 hours to 12 hours to allow the device to be incorporated more easily into a patient's regular daily routine. During the last 5 days of the 14-day device usage period, women were supplied with pre-weighed pads and instructed to wear them for 12 hours each day while using the device and to record the number and type of incontinence episodes experienced during each 12-hour period. Patients did not use the intrav- 
aginal device during menstruation, but resumed use after cessation of menses so that a total of 14 days were achieved. The device was also removed before sexual intercourse. Pads were collected and weighed at each visit as an indication of unintentional urine loss. Pads had been placed inside ziplock bags and frozen until the study visit to avoid any loss of weight through evaporation. The patient was also instructed to record the number and type of incontinence episodes experienced during each 12-hour period of wear. Adverse events were recorded throughout the study.

Patients completed a daily diary entry regarding device characteristics. Data collected included time of device insertion and removal; discomfort with insertion, removal, or wearing the device; and whether there was any spotting on the device after removal. Overall comfort with the device was scored daily by each patient, with $1=$ very uncomfortable and $5=$ very comfortable.

Adverse eventss were reported by patients and/or detect by the investigator or other site personnel. Causality with regard to the investigational product was determined by whether or not medical evidence existed to suggest that the $\mathrm{AE}$ was related to usage of the investigational product or whether there was a more probable explanation.

At the completion of 14 days of device usage, an exit visit was conducted at which both QoL assessments were repeated as were all safety assessments (including gynecological exams, vaginal swabs, UTI culture, and a general physical examination).

Efficacy analyses were based on a comparison of the results from the 5-day baseline period to the last 5 days of the 14-day device usage period. Statistical analyses were conducted using PC SAS (Releases 8.2 or 9.1.3). Statistical significance was declared at the two-sided 0.05 significance level. No adjustments were made for multiple endpoints. Safety data were analyzed using the intent-to-treat (ITT) population defined as all patients who used the device at least once during the study. Data from physical examinations, gynecological assessments, and urinalysis results were summarized with the appropriate descriptive statistics (e.g., number and percentage of patients, mean, standard deviation) overall and by device size and visit. All AEs were summarized by severity and causality.

Efficacy data were summarized using the per-protocol (PP) population, defined as the subset of patients who met all protocol inclusion/exclusion criteria and were compliant with the study protocol. The primary efficacy endpoint was the reduction in SUI episodes from the 5-day baseline period to the last 5 days of the 14-day device usage period based on patient diaries and questionnaires. The difference between the study periods in the number of SUI episodes was to be analyzed using a paired t-test for normally distributed data or a nonparametric analysis (sign test, sign-rank test, or Cochran-Mantel-Haenszel test depending on the distribution of the data) for data that were not normally distributed.

The secondary efficacy endpoint was the reduction in PWG from the 5-day baseline period to the last 5 days of the 14-day device usage period. Pad weight gain was normalized by wear time prior to any other calculations to give the average urine loss per hour. The change in PWG was determined by subtracting the average normalized PWG of the 5-day baseline period from the average normalized PWG of the last 5 days of the 14-day device usage period. The difference between the study periods in the amount of PWG was analyzed in the same manner described above for the primary endpoint.

Quality of life data were summarized using the PP population. All item responses from the IIQ-7 and the PPBC questionnaires were summarized by device size and visit. Changes in item responses from Visit 1 (screening/baseline) to Visit 4 (exit) were analyzed using the Cochran-MantelHaenszel test.

Sample size was not based on statistical considerations of power for comparative inference. However, it was assumed, based on a standard deviation of 6 , a sample size of 50 , and normal and $t$ distribution approximations, that there would be approximately $93 \%$ power to detect a $50 \%$ reduction in SUI episodes using a paired $t$ test.

\section{RESULTS}

Fifty-seven patients were enrolled and included in the ITT population. Of these, five patients withdrew due to discomfort with the device. Four other patients were excluded from the PP population because their diaries documented urge incontinence instead of SUI (despite the fact that SUI had been self-reported at baseline). Therefore, the PP population was comprised of 48 patients.

The mean age of the study population was 48 years and most $(93 \%)$ were Caucasian. Approximately half of the patients were premenopausal at baseline. Of the patients who were premenopausal, $29 \%$ had undergone hysterectomies, whereas $76 \%$ of postmenopausal patients were hysterectomized.

\section{Safety Results}

There were no significant clinical changes in patients' gynecological examinations after 2 weeks of daily device use as recorded by the investigators. Shifts in vaginal $\mathrm{pH}$ were observed during the study; however, the clinical interpretation of these shifts is unclear in this mixed population of preand post-menopausal women. Women with higher and lower $\mathrm{pH}$ values at baseline shifted towards the baseline mean of 4.5 after device usage. A total of five patients began the study with a vaginal $\mathrm{pH}$ of 5.5 and five had this value at the end of the study.

There were no clinically significant changes from baseline with regard to gynecological examinations or urinalysis at the end of the study. Dipstick urinalysis results revealed that one patient was positive for esterase at baseline (negative at exit) and four patients were positive for esterase at exit (negative at baseline). No specimens were positive for nitrites. One clinical diagnosis of UTI was made during the study and reported as an AE. This patient had normal baseline and exit urinalysis results. These results provide evidence that use of the device was not associated with an increased incidence of urinary tract infection.

Fifteen of the patients with normal cervical and/or vaginal cytopathology at baseline had abnormal results reported at the exit visit (11 out of 15 had atypical squamous cells of undetermined significance [ASCUS]). Nine of these 15 patients (including one patient with "high grade" changes) had 
human papillomavirus (HPV) testing done as part of the Pap smear procedure and all results were negative. Follow-up cytologic results were normal in all patients who underwent a follow-up procedure (11 out of 11 patients).

Mean comfort score during the fitting period was 3.27, improving slightly to 3.49 during device usage.

In general, the TIPI intravaginal device was well tolerated, and only 5 of the 57 patients initially enrolled in the study withdrew after trying only a few devices. These patients reported discomfort inserting, wearing, or removing the device as the reason for withdrawal. Tolerability of the device was further assessed by in-depth, one-on-one interviews in a parallel study that will be described in a subsequent report.

Compliance with device usage instructions was acceptable in that $54(96.4 \%)$ women wore the device for the suggested length of time (12 hours \pm 1 hour), as recorded in a daily device usage diary. Of 755 devices for which weartime data were available, $712(94.3 \%)$ were used for 12 hours \pm 1 hour.

Patients were also asked to write "yes" or "no" to the following question on the daily device usage diary: "Spotting on Device?" Of the 57 ITT patients, 38 (67\%) noted spotting on at least one of the devices. Eighteen (32\%) patients noted no spotting on any of the devices, $29(51 \%)$ noted spotting on $1 \%$ to $50 \%$ of the devices, and $9(16 \%)$ noted spotting on $>50 \%$ of the devices. The observation of "spotting on device" should be distinguished from the clinical occurrence of vaginal spotting. Only one patient complained of vaginal spotting during the period of device usage, which was reported as an AE. Two other patients complained of "spotting on device" which were reported as AEs. Both of these patients reported using 14 devices during the study and recorded spotting on 12 of these devices. The observation of "spotting on device" thus appears to be a finding that was elicited by including it on the daily device usage diary and should not be confused with the observation of clinical "spotting."

Twenty- three $(40 \%)$ patients who reported a total of 38 AEs during the study; $53 \%$ of these were considered definitely not related to the device by the investigator. Of the 18 AEs considered potentially related to device usage, $3(8 \%)$ were considered doubtfully related, $5(13 \%)$ possibly related, and $10(26 \%)$ were considered probably related. The AEs considered possibly or probably related included vaginal spotting, vaginal irritation, a sense of lower pelvic pressure, cramping, headache, and nausea, none of which required any intervention. Three patients had abnormal pap smears. Subsequent HPV tests were negative, and follow-up Pap smears were normal. Of all reported AEs, $36(95 \%)$ were mild in severity, and $2(5 \%)$ were reported as moderate; no serious AEs were reported.

\section{Efficacy Results}

Efficacy data were summarized using the PP population; however, one patient who had SUI data (from daily diary entries) was missing PWG data, and one patient who had PWG data was missing SUI data from the diaries. Missing data points were not imputed in the primary analysis.

A reduction in SUI episodes was observed when the 5day baseline period was compared to the last 5 days of the 14-day device usage period, based on daily diaries. After less than 2 weeks of daily device use, SUI episodes during the 5day period of observation were reduced by $78 \%$ compared to the 5-day baseline period for patients in the PP analysis.. Twenty-nine percent of patients had at least a $90 \%$ reduction in SUI episodes, $25 \%$ had an $80 \%$ to $90 \%$ reduction, $12.5 \%$ had a $70 \%$ to $79 \%$ reduction, and $8.3 \%$ had a $60 \%$ to $69 \%$ reduction. Overall, $75 \%$ of patients had at least a $60 \%$ reduction in SUI episodes during the 5-day observation period of device use (Table 1).

A statistically significant decrease in the number of SUI episodes was also observed from the 5-day baseline period (median: 13.5 episodes/5-day period [inter-quartile range: $10.0,18.5])$ to the last 5 days of the 14-day device usage period (median: 3.0 episodes/5-day period [inter-quartile range: $1.0,7.0])(P \leq 0.001)$.

A reduction in daily PWG was also observed when the 5day baseline period was compared to the last 5 days of the 14-day device usage period. A statistically significant decrease in daily PWG was observed from the baseline period (median: $1.5 \mathrm{~g} / 12$-hour wear period [inter-quartile range: $0.40,3.3])$ to the last 5 days of the 14-day device usage period (median: $0.22 \mathrm{~g} / 12$-hour wear period [inter-quartile range: $0.09,0.60])(P \leq 0.0001)$.

The subjective perspective of patients with regard to the severity of their incontinence also improved with device usage, as assessed by the PPBC questionnaire. A statistically

Table 1. Summary of the Percent Reduction in SUI Episodes (Per-protocol Patients $[N=48]$ )

\begin{tabular}{|c|c|c|c|c|}
\hline & \multicolumn{3}{|c|}{ Device Size } & $\begin{array}{c}\text { Total } \\
(\mathbf{n = 4 8})\end{array}$ \\
\hline $\begin{array}{c}\text { Percent Reduction in SUI } \\
\text { Episodes }\end{array}$ & $\begin{array}{c}\mathbf{1} \\
(\mathbf{n = 2 0})\end{array}$ & $\begin{array}{c}\mathbf{3} \\
(\mathbf{n = 2 6})\end{array}$ & 0 & $12(25.0)$ \\
\hline \hline$\leq 59$ & $5(10.4)$ & $7(14.6)$ & $1(2.1)$ & $4(8.3)$ \\
\hline $60-69$ & $2(4.2)$ & $1(2.1)$ & 0 & $6(12.5)$ \\
\hline $70-79$ & $3(6.3)$ & $3(6.3)$ & 0 & $12(25.0)$ \\
\hline $80-89$ & $5(10.4)$ & $7(14.6)$ & $1(2.1)$ & $14(29.2)$ \\
\hline
\end{tabular}

Data shown are $\mathrm{n}(\%)$ of patients.

$\mathrm{SUI}=$ stress urinary incontinence. 
Table2. Summary of the Number and Percentage of Patients Who Reported Moderate to Many Severe Bladder Problems (Per-protocol Patients $[\mathrm{N}=48]$ )

\begin{tabular}{|c|c|c|c|}
\hline & $\begin{array}{l}\text { Visit } 1 \\
\text { n(\%) }\end{array}$ & $\begin{array}{l}\text { Visit } 4 \\
\text { n(\%) }\end{array}$ & $P$-value ${ }^{a}$ \\
\hline All device sizes $(n=48)$ & $36(75.0)$ & $18(37.5)$ & $<0.001$ \\
\hline Device size $1(\mathrm{n}=20)$ & $14(70.0)$ & $8(40.0)$ & 0.014 \\
\hline Device size $2(\mathrm{n}=26)$ & $20(76.9)$ & $9(34.6)$ & 0.002 \\
\hline Device size $3(n=2)$ & $2(100)$ & $1(50.0)$ & - \\
\hline
\end{tabular}

Possible responses to the Patient Perception of Bladder Condition (PPBC) included the following: does not cause me any problems at all; causes me some very minor problems; causes me some minor problems; causes me (some) moderate problems; causes me severe problems; causes me many severe problems.

${ }^{\mathrm{a}} \mathrm{P}$-values are based on Cochran-Mantel-Haenszel.

significant decrease in the overall percentage of patients who reported moderate bladder problems or worse was observed from baseline at Visit 1 (75\% of patients) to exit at Visit 4 (37.5\% of patients) $(P<0.001)$ based on the PP population (Table 2).

Use of the device also produced substantial improvement in the patients' perceived quality of life as assessed by the IIQ-7 questionnaire. In addition, based on the PPBC questionnaire, the percentage of patients who reported moderate to very severe bladder problems significantly decreased as compared to baseline.

All seven areas evaluated by the IIQ-7 demonstrated an improvement in the quality of life. Statistically significant decreases in the percentage of patients who reported moderate or severe impact of SUI after device usage, however, were seen only with regard to feeling frustrated $(\mathrm{p}=0.011)$, impact on social activities $(p=0.018)$, and impact on recreational activities $(\mathrm{p}=0.001)$. A non-significant decrease was observed in the percentage of patients who felt that incontinence impacted entertainment activities $(\mathrm{p}=0.052)$ (Table 3 ).

\section{DISCUSSION AND CONCLUSIONS}

Stress urinary incontinence is a disorder that affects a substantial number of older women, and one that carries a significant burden with regards to daily activities and overall quality of life. Many of women with SUI are poor surgical candidates.

Pessary use is a potentially useful nonsurgical option. Use of vaginal pessaries dates back before the time of Hippocrates [9] as a way to support the uterus and or bladder [10]. In an ongoing attempt to improve efficacy as well as comfort of wear, 123 kinds of pessaries had been developed by 1867 [10].

Traditionally, however, use of the pessary has not been widely embraced. Pessaries have for the most part been uncomfortable and tedious to manage. They have also been associated with the idea that pessaries are at best an antiquated therapy and at worst, potentially dangerous [11]. Christ and Haja [12], in a 1978 study of cytopathological changes associated with vaginal pessary use, observed that long-term pessary use was "invariably accompanied by trauma to the atrophic vaginal epithelium of the postmenopausal woman," and that the cytologic changes "observed in association with pessary use reflect the mechanical trauma of the pessary and the resultant inflammatory and reparative response."

Most physicians, however, even in the fields of gynecology and urogynecology, have very little training on pessaries

Table 3. Summary of the Number and Percentage of Patients Who Reported Moderate or Severe Impact of SUI on Each Item of the IIQ-7 (Per-protocol Patients $[N=48]$ )

\begin{tabular}{|c|c|c|c|}
\hline IIQ-7 Item ${ }^{a}$ & $\begin{array}{l}\text { Visit } 1 \\
\text { n(\%) }\end{array}$ & $\begin{array}{l}\text { Visit } 4 \\
\text { n(\%) }\end{array}$ & $P$-value ${ }^{\mathrm{b}}$ \\
\hline Household chores & $17(35.4)$ & $12(25.0)$ & 0.225 \\
\hline Emotional health & $16(33.3)$ & $12(25.0)$ & 0.248 \\
\hline Entertainment activities & $20(41.7)$ & $13(27.1)$ & 0.052 \\
\hline Feeling frustrated & $27(56.3)$ & $19(39.6)$ & 0.011 \\
\hline Social activities & $25(52.1)$ & $15(31.3)$ & 0.018 \\
\hline Ability to travel ${ }^{c}$ & $20(42.6)$ & $16(34.0)$ & 0.248 \\
\hline Recreational activities & $33(68.8)$ & $19(39.6)$ & 0.001 \\
\hline
\end{tabular}

IIQ-7= Incontinence Impact Questionnaire, SUI = stress urinary incontinence.

atem responses were assigned values of 0 for "not at all," 1 for "slightly," 2 for "moderately," and 3 for "greatly."

${ }^{\mathrm{b}} P$-values are based on Cochran-Mantel-Haenszel equivalent of McNemar's test.

${ }^{\mathrm{c}}$ One patient did not answer this question at Visit 4. 
or their use [11]. Interest in pessaries as a reasonable firstline therapy in women with pelvic-floor support issues is now on the rise, and pessaries are enjoying a resurgence in design and novel applications for use [13].

This study confirmed, in a real-use context, previously reported studies [7,8] which demonstrated that in women with predominantly SUI, the TIPI intravaginal device was effective, well-tolerated, and improved QoL. Use of the device was also shown to have a good safety profile. Only $39 \%$ percent of AEs observed were considered to be either possibly or probably related device usage and $95 \%$ of those were mild. No serious AEs were recorded. Patients in this study had baseline and exit Pap Smears. Fifteen patients were found, at study end, to have abnormal Pap smear results. Follow-up Pap Smears results were normal in all patients. The rapidity of the observed changes (from normal to abnormal may suggests that these changes may have been artifactual. Indeed, some authorities suggest that vaginal lubricants, vaginal douching, or tampon use can cause artifactual results [14-16]. These factors were not controlled, in this study, before taking PAP smears from the patients. A learning that should be considered in any future studies.

This paper presents an alternative therapy for urinary incontinence that appears to be not only effective, well tolerated but is also noninvasive and disposable. The TIPI is a new disposable device which can be self-inserted into the vagina on a daily basis. This disposable device offers a potential advantage over existing incontinence pessaries in that traditional pessaries largely remain in place for much prolonged periods and could have the potential to be associated with vaginal erosion [17], foul odors, infections, and irritation [18]. In contrast, the TIPI device during usage was given a mean comfort score of 3.49 on a five-point scale, with 91.2 $\%$ of patients maintaining use. (Only around 8.8\% terminated use because of discomfort). Pessaries that succeed in providing both comfort and control of incontinence represent an attractive substitute for the more invasive treatments for SUI.

Our current study, unlike the two previous studies, evaluated the use of the device for up to 12 hours a day, for 14 days of device wear, in subjects. These preliminary results suggest that the TIPI may be a useful nonsurgical alternative to current therapies for SUI. Under the conditions of this study, the device was well-tolerated, easy to use, avoids the more tedious and expensive medical management required for traditional incontinence pessaries, and appears to be an effective therapy. Long-term data would be more fully able to evaluate long-term tolerability and safety as well as whether use of the TIPI could replace surgical intervention.

\section{ACKNOWLEDGEMENT}

The authors would like to thank Ms. Michaelle Jones for statistical analysis; Ms. Cindy Springer for being the Clinical Trial Manager, Dr. Elan Ziv for technical review, Ms. Ze- inab Schwen and Ms. Wendy Wippel (Strategic Regulatory Consulting, Cincinnati, $\mathrm{OH}$ ) for their assistance in the preparation of this manuscript.

\section{CONFLICTS OF INTEREST}

Declared none.

\section{REFERENCES}

[1] Diokno AC, Estanol MVC, Ibrahim IA, Balasubramaniam M Prevalence of urinary incontinence in community dwelling men: a cross sectional nationwide epidemiological survey. Int Urol Nephrol 2007; 39: 129-36

[2] Harrison GL, Memel DS. Urinary incontinence in women: its prevalence and its management in a health promotion clinic. $\mathrm{Br} \mathbf{J}$ Gen Pract 1994; 44: 149-52.

[3] Nygaard I, Barber MD, Burgio KL, et al. Prevalence of symptomatic pelvic floor disorders in US women. JAMA 2008; 300: 13116.

[4] Hu T, Wagner TH, Bentkover JD, Leblanc K, Zhou SZ, Hunt T. Costs of urinary incontinence and overactive bladder in the United States: a comparative study. Urology 2004; 63: 461-5.

[5] Basu M, Duckett JRA. Update on duloxetine for the management of stress urinary incontinence. Clin Interv Aging 2009; 4: 25-30.

[6] Luber KM. The definition, prevalence, and risk factors for stress urinary incontinence. Rev Urol 2004; 6 (Suppl 3): S3.

[7] Ziv E, Stanton SL, Abarbanel J. Efficacy and safety of a novel disposable intravaginal device for treating stress urinary incontinence. Am J Obstet Gynecol 2008; 198: 594 e1-7.

[8] Ziv E, Stanton SL, Abarbanel J. Significant improvement in the quality of life in women treated with a novel disposable intravaginal device for stress urinary incontinence. Int Urogynecol J Pelvic Floor Dysfunct 2009; 20: 651-8.

[9] Bash KL. Review of vaginal pessaries. Obstet Gynecol Surv 2000; 55: 455-60.

[10] Zeitlin MP, Lebherz TB. Pessaries in the geriatric patient. J Am Geriatr Soc 1992; 40: 635-9.

[11] Cundiff GW, Weidner AC, Visco AG, Bump RC, Addison WA. A survey of pessary use by members of the American Urogynecologic Society. Obstet Gynecol 2000; 95: 931-5.

[12] Christ ML, Haja J. Cytologic changes associated with vaginal pessary use. With special reference to the presence of Actinomyces. Acta Cytol 1978; 22: 146-9.

[13] Hanson LM, Schulz JA, Flood CG, Cooley B, Tam F. Vaginal pessaries in managing women with pelvic organ prolapse and urinary incontinence: Patient characteristics and factors contributing to success. Int Urogynecol J Pelvic Floor Dysfunct 2006; 17: 1559.

[14] Arbyn M, De Cock R. Flemish Steering Group on Cervical Cancer Screening. A technical guideline: collection of adequate pap smears of the uterine cervix. Brussels: Scientific Institute for Public Health 2001.

[15] Ball C, Madden JE. Update on cervical cancer screening. Current diagnostic and evidence-based management protocols. Postgrad Med 2003; 113: 59-64.

[16] Hong Kong Society of Cytology. Cervical cytology practice guidelines 2002.

[17] Adams E, Thomson A, Maher C, Hagen S. Mechanical devices for pelvic organ prolapse in women. Cochrane Database Syst Rev 2004; Doi: 10.1002/14651858: CD004010.

[18] Bai SW, Yoon BS, Kwon JY, Shin JS, Kim SK, Park KH. Survey of the characteristics and satisfaction degree of the patients using a pessary. Int Urogynecol J Pelvic Floor Dysfunct 2005; 16: 182-6. 